Adam Linder James A. Russell

\section{An exciting candidate therapy for sepsis: ulinastatin, a urinary protease inhibitor}

Received: 4 June 2014

Accepted: 6 June 2014

Published online: 3 July 2014

(c) Springer-Verlag Berlin Heidelberg and ESICM 2014
A. Linder · J. A. Russell ( $\square)$

Centre for Heart Lung Innovation, St. Paul's Hospital, University of British Columbia, 1081 Burrard Street, Vancouver, BC V6Z 1Y6, Canada

e-mail: jrussell@mrl.ubc.ca; Jim.Russell@hli.ubc.ca

\section{J. A. Russell}

Division of Critical Care Medicine, St. Paul's Hospital, University of British Columbia, 1081 Burrard Street, Vancouver, BC V6Z 1Y6, Canada
In a recent issue of Intensive Care Medicine (ICM), Karnad et al. [1] reported a randomized, double-blind, placebo-controlled multicenter trial (RCT) evaluating the efficacy of a urinary protease inhibitor (ulinastatin) compared to placebo in patients with severe sepsis. This RCT was conducted in the intensive care units (ICUs) of seven tertiary care hospitals in India including 114 patients. Patients received intravenous ulinastatin or placebo twice daily for 5 days. Ulinastatin was associated with significantly decreased mortality (7.3 vs. $20.3 \%$, $P=0.042$ ), with $25 \%$ of the deaths in the ulinastatin group judged related to ARDS compared to $42 \%$ in the placebo group, lower frequency of new organ dysfunction, and shorter durations of mechanical ventilation and hospital stay compared to placebo. Furthermore, in stepwise multiple logistic regression (adjusted for age, gender, GCS, specific organ failures, number of organ failures, need for vasopressor, and mechanical ventilation), treatment with ulinastatin was associated with a statistically significant decrease in mortality.

This is an interesting trial with quite impressive results given the relatively small sample size, which raises these questions: what is ulinastatin and why might it be important in sepsis?

Ulinastatin (UTI) is a multifunctional Kunitz-type serine protease inhibitor found in human urine and blood. UTI (also known as ulinastatin, HI-30, ASPI, or bikunin) is produced by hepatocytes and belongs to a group of proteins known as the inter- $\alpha$-inhibitor (I $\alpha \mathrm{I})$ family. During inflammation, ulinastatin is cleaved from $\mathrm{I} \alpha \mathrm{I}$ family proteins through proteolytic cleavage by neutrophil elastase in the peripheral circulation or at sites of inflammation. Ulinastatin inhibits various serine proteases that are important in the pathophysiology of sepsis including trypsin, thrombin, chymotrypsin, kallikrein, plasmin, elastase, cathepsin, and factors IXa, Xa, XIa, and XIIa. Ulinastatin also inhibits inflammation by suppressing the infiltration of neutrophils and release of elastase and inflammatory mediators from neutrophils. Ulinastatin also inhibits the production of TNF- $\alpha$, IL- 1 , and IL- 6 possibly through suppression of MAPK signalling pathway [2].

The RCT of Karnad and colleagues in ICM supports previous clinical trials of ulinastatin (Table 1). A recent systematic review and meta-analysis found 29 RCTs of ulinastatin in ARDS with more than 1,700 participants. Even though the authors concluded that most studies were of poor quality they found that ulinastatin decreased ICU mortality, improved oxygenation, and decreased duration of hospital stay [3]. Clinically ulinastatin is already in use, for instance in Japan, to treat acute pancreatitis (postendoscopic retrograde cholangiopancreatography pancreatitis), in which proteases play a pathophysiological role. Other clinical conditions for which ulinastatin has been evaluated clinically are "systemic inflammatory response 


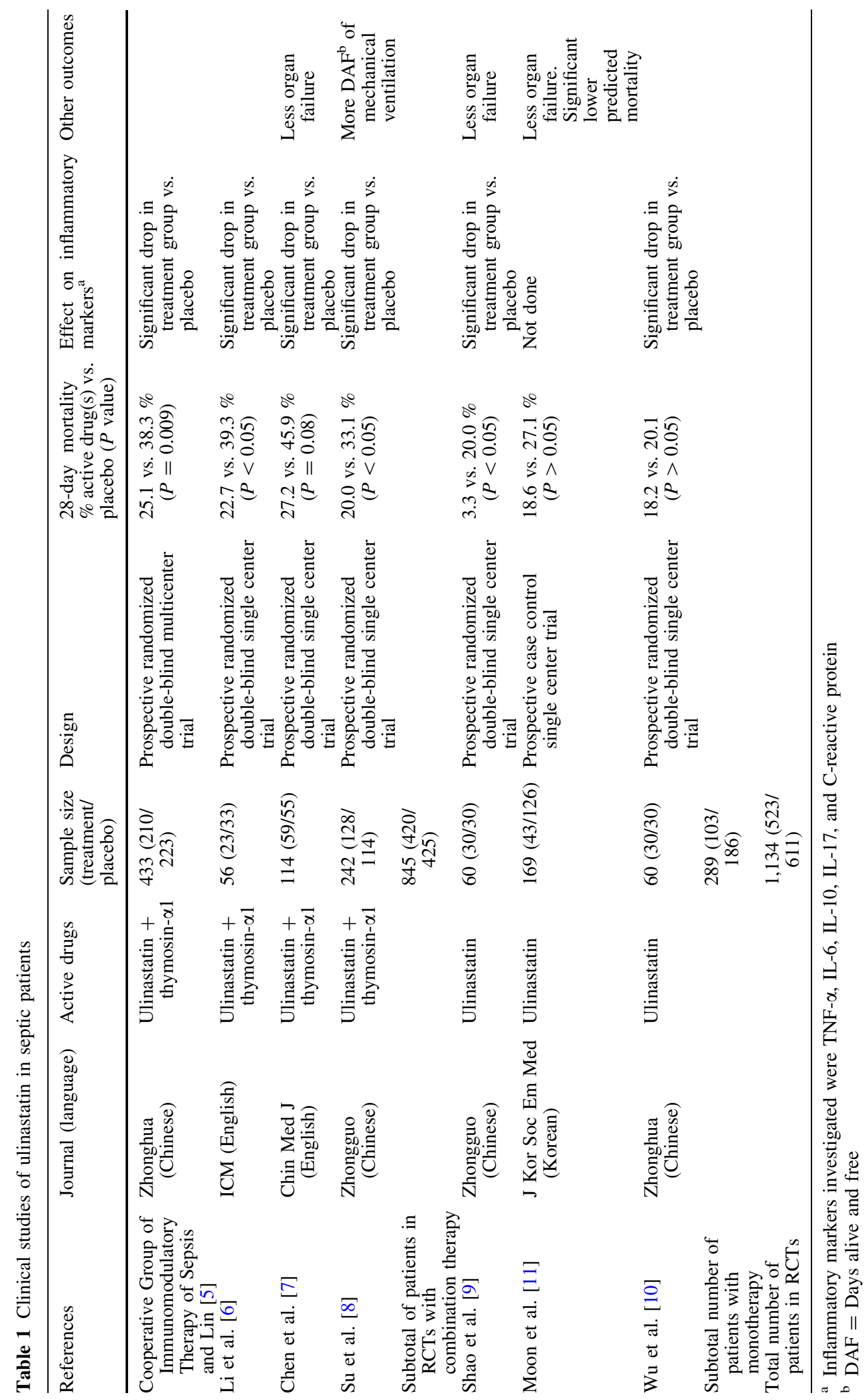


syndrome" conditions such as burns, post cardiac surgery, and ARDS (e.g., in China).

Protease inhibitors are used to treat many different medical conditions, such as cancer and Alzheimer's disease. In cancer treatment, there is still a widely held view that "broad spectrum" protease inhibitors will be needed to treat malignancies at particular stages [4].

This approach-using a more semi-selective antagonist - has not so far been widely adopted in treatment of sepsis. Ulinastatin has been evaluated as a sepsis therapeutic both as a single drug and in combination with the immunomodulatory agent thymosin- $\alpha 1$ (Table 1 ). We identified seven RCTs of ulinastatin in septic patients [511]. All these RCTs showed benefit of ulinastatin such as significant improvement in inflammatory markers and, to a lesser extent, in organ dysfunction. All studies showed lower 28-day mortality in the ulinastatin treatment arm. However, the majority of patients (more than 800) were treated with ulinastatin in combination with thymosin- $\alpha 1$ making interpretation of the independent therapeutic potency of ulinastatin difficult.

Ulinastatin and members of the I $\alpha$ I family are also considered as candidate biomarkers for diagnosis and prognosis of sepsis [12], but for some reason no clinical studies appear to have been done in Western countries to date. A clintrials.gov trial search revealed nine registered studies with ulinastatin, mainly focusing on cardiac surgery and ARDS, and none on sepsis treatment.

However, even if urinary protease inhibitors have biological plausibility for the treatment of sepsis, there are still a number of concerns of which many are highlighted by the comments from Saigal and Kapoor [13]. These include the high exclusion rate, young age, the higher presence of multidrug resistance in the control group, and the high mortality rate of the control group that mitigate the generalizability of the results to other countries. Also, the mean length of stay of 26.2 days for a 37-year-old man with one organ dysfunction $(64 \%)$ is strikingly high. Sample size calculation was performed assuming a 28-day all-cause mortality of $30 \%$ in the control group and $10 \%$ in the study group. A sample size of 59 completed patients in each group was required to attain a power of $80 \%$ at significance level of $5 \%$. This is an extraordinarily high effect size and completely different from any prior RCT in severe sepsis. The trial cohort was very young (mean age, 37 years; mean age is about 55 years in most sepsis RCTs). The surprisingly low APACHE II could be well explained in part by young age. Still, we suggest that the mortality rate is high for severe sepsis in the control group given the mean age of 37 years, low rate of positive cultures (15/59), and predominance $(65 \%)$ of patients who had only one organ dysfunction. There is no clear explanation for this and thus there are concerns regarding the generalizability of this RCT, which highlights the need for further larger RCTs of ulinastatin in North America, Europe, and Australasia.

There are other "broad spectrum" protease inhibitors already available on the market, such as aprotinin (Trasylol), which have not been tested in the context of human sepsis treatment. It is not clear whether ulinastatin has advantages over such other available protease inhibitors.

What are the research and clinical implications of this publication? In conclusion, this is a provocative but not yet convincing RCT of ulinastatin in severe sepsis. It will be critical to see further preclinical evaluation of the mechanism(s) of action, comparison to other available protease inhibitors, and to see large well-conducted RCTs of ulinastatin in other countries.

Conflicts of interest None.

\section{References}

1. Karnad DR, Bhadade R, Verma PK, Moulick ND, Daga MK, Chafekar ND, Iyer S (2014) Intravenous administration of ulinastatin (human urinary trypsin inhibitor) in severe sepsis: a multicenter randomized controlled study. Intensive Care Med 40:830-838. doi:10.1007/s00134014-3278-8

2. Inoue K, Takano H (2010) Urinary trypsin inhibitor as a therapeutic option for endotoxin-related inflammatory disorders. Expert Opin Investig Drugs 19(4):513-520
3. Leng YX, Yang SG, Song YH, Zhu X, Yao GQ (2014) Ulinastatin for acute lung injury and acute respiratory distress syndrome: a systematic review and meta-analysis. World J Crit Care Med 3(1):34-41

4. Smyth E (2003) The trouble with inhibitors. Nature Publishing Group, Horizon Symposia

5. Cooperative Group of Immunomodulatory Therapy of Sepsis, Lin HY (2007) Clinical trial with a new immunomodulatory strategy: treatment of severe sepsis with ulinastatin and maipuxin. Zhonghua Yi Xue Za Zhi 87(7):451-457
6. Li Y, Chen H, Li X, Zhou W, He M, Chiriva-Internati M, Wachtel MS, Frezza EE (2009) A new immunomodulatory therapy for severe sepsis: ulinastatin plus thymosin alpha 1. J Intensive Care Med 24(1):47-53

7. Chen H, He MY, Li YM (2009)

Treatment of patients with severe sepsis using ulinastatin and thymosin alpha1: a prospective, randomized, controlled pilot study. Chin Med J 122(8):883-888

8. Su L, Meng FS, Tang YQ, Wen Q, Liu YS, Tang LQ, Duan PK, Luo RJ (2009) Clinical effects of ulinastatin and thymosin alpha1 on immunemodulation in septic patients. Zhongguo Wei Zhong Bing Ji Jiu Yi Xue 21(3):147-150 
9. Shao YM, Zhang LQ, Deng LH, Yao HG (2005) Clinical study on effects of ulinastatin on patients with systemic inflammatory response syndrome. Zhongguo Wei Zhong Bing Ji Jiu Yi Xue 17(4):228-230

10. Wu TJ, Zhang LN, Kang CC (2013) The effect of ulinastatin on disbalance of inflammation and immune status in patients with severe sepsis. Zhonghua Wei Zhong Bing Ji Jiu Yi Xue 25(4):219-223
11. Moon SW, Lee SW, Hong YS, Park DW, Jang IJ, Yoon YH, Lim SI (2009) The effects of urinary trypsin inhibitor on the outcomes of severe sepsis and septic shock patients. J Korean Soc Emerg Med 20:80-85

12. Opal SM, Lim YP, Siryaporn E, Moldawer LL, Pribble JP, Palardy JE, Souza S (2007) Longitudinal studies of inter-alpha inhibitor proteins in severely septic patients: a potential clinical marker and mediator of severe sepsis. Crit Care Med 35(2):387-392
13. Saigal S, Kapoor G (2014) Ulinastatin: is it worth using in severe sepsis? Intensive Care Med. doi:10.1007/ s00134-014-3341-5 